\title{
Adult-onset Still's disease preceded by influenza vaccination: Coincidence or true association?
}

\author{
Kanon Jatuworapruk
}

\begin{abstract}
Background: Influenza vaccine has rarely been associated with the occurrence of systemic inflammatory diseases.

Objective: To present a case of adult-onset Still's disease (AOSD) following influenza vaccination, and subsequently explore the possible association between AOSD and influenza vaccination, as well as implications for clinical practice.

Methods: Case report.

Results: We report a case of 20-year-old woman who developed typical AOSD, characterized by spiking fever, arthritis and salmon-pink rash, one week after influenza vaccination. She responded well to systemic corticosteroids therapy. This is the first case report of influenza vaccination-associated AOSD from Thailand.

Conclusion: Influenza vaccination may be a trigger of AOSD but more data are needed to confirm the association. History of recent vaccination should be explored in patients suspected of having systemic inflammatory diseases including AOSD.
\end{abstract}

Key words: adult-onset Still's disease, influenza vaccination, fever of unknown origin, arthritis, pneumonitis

\section{From:}

Division of Rheumatology, Department of Medicine,

Faculty of Medicine, Thammasat University, Pathumthani, Thailand

\section{Corresponding author:}

Kanon Jatuworapruk

Department of Medicine, Faculty of Medicine, Thammasat University, 99/209 Paholyotin Road, Khlong Luang, Pathumthani, 12120 Thailand E-mail: kanon@tu.ac.th

vaccine over a decade ago., ${ }^{7,8}$ We hereby report a case of a young woman who developed AOSD after receiving an influenza vaccine. To the best of our knowledge, this is the third of such case and the first from Thailand.

\section{Report of case}

The patient was a 20-year-old woman of Burmese descent, who worked in a factory and has previously been in good health. Three weeks before hospital admission, she received an intramuscular trivalent influenza vaccine from a community hospital. Seven days after the vaccination, she reported having fever which usually worsened in the evening, as well as generalized myalgia and mild tension-type headache. She took two tablets of acetaminophen $(1000 \mathrm{mg})$ daily for a week but the fever persisted. One week before admission, she developed bilateral knee and ankle pain which lead to limited weight bearing of the lower extremities. She visited an outpatient clinic at a general hospital where she was started previous reports of presumptive AOSD triggered by influenza 
on oral amoxicillin (2 gm daily) and underwent blood tests: negative dengue IgG, IgM and NS1 antigen, hemoglobin 11.0 $\mathrm{g} / \mathrm{dL}$, hematocrit $32.2 \%$, white blood cells (WBC) 19,990 cells/ $\mathrm{mm}^{3}$ (neutrophil $94 \%$ ), platelet 219,000 cells $/ \mathrm{mm}^{3}$ and normal urine analysis. Her symptoms did not improve after seven-day course of amoxicillin. She subsequently came to our hospital where she was hospitalized.

Daily high spiking fever $\left(>39^{\circ} \mathrm{C}\right)$ was recorded throughout her three-week hospital course, with spikes typically present in late mornings and late afternoons (figure 1). In addition to fever, three episodes of self-limiting maculopapular salmon-pink rash on the back and upper chest were observed, with each episode lasting between three to six hours (figure 2). Physical examinations showed normal blood pressure, tachycardia corresponding to fever spikes, mild tenderness with no swelling of the knees and ankle joints, no palpable lymph nodes, normal chest auscultation and no hepatic or splenic enlargement. The patient received the following empirical antibiotics starting on the first day of admission without any clinical response: ceftriaxone (2 gm daily) for seven days, doxycycline (200 mg daily) for seven days and azithromycin (500 mg daily) for three days.

Extensive investigation was pursued in order to exclude systemic autoimmune disease, occult malignancy and infection. Basic laboratory results were unremarkable except for the neutrophil-dominant leukocytosis and mild transaminitis: hemoglobin $11.0 \mathrm{~g} / \mathrm{dL}$, hematocrit $34.5 \%$, WBC 15,100 cells/ $\mathrm{mm}^{3}$ (neutrophil $84 \%$ ), platelet 352,000 cells $/ \mathrm{mm}^{3}$, creatinine $0.5 \mathrm{mg} / \mathrm{dL}$, serum albumin $3.3 \mathrm{~g} / \mathrm{dL}$, aspartate transaminase (AST) $198 \mathrm{U} / \mathrm{L}$, alanine transaminase (ALT) $81 \mathrm{U} / \mathrm{L}$, alkaline phosphatase $93 \mathrm{U} / \mathrm{L}$, creatinine phosphokinase $(\mathrm{CPK}) 52$ $\mathrm{U} / \mathrm{L}$, normal thyroid function, blood sugar $99.6 \mathrm{mg} / \mathrm{dL}$, and normal urine analysis. Serum ferritin were markedly elevated at $>15,000 \mathrm{ng} / \mathrm{mL}$ (normal range 10-120 ng/mL). Tests for possible infections yielded all negative results: negative serum IgM and IgG antibodies against chikungunya virus, scrub and murine typhus, non-reactive TPHA and rapid plasma reagins for syphilis, negative malaria think and thin films

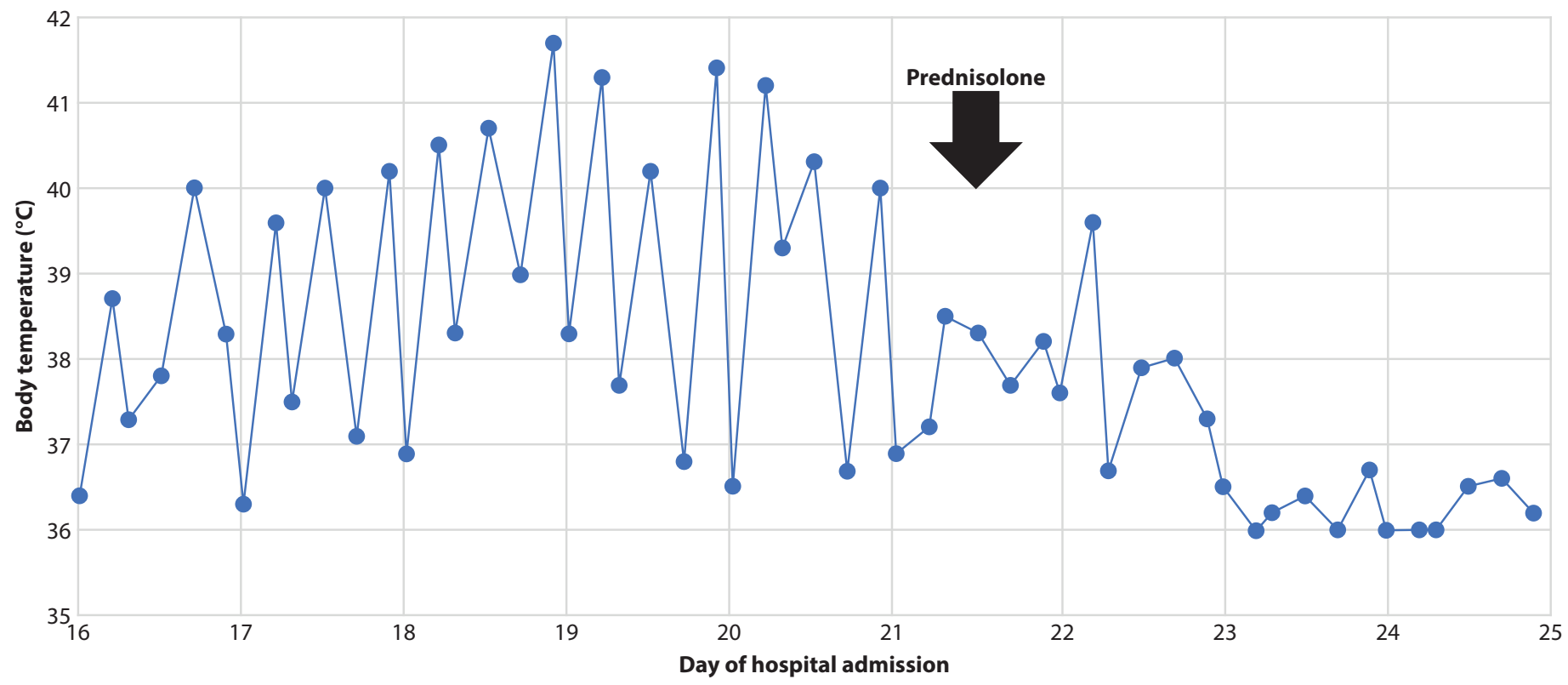

Figure 1. Fever pattern in the last 10 days of hospitalization. Arrow indicates the start of corticosteroids therapy.

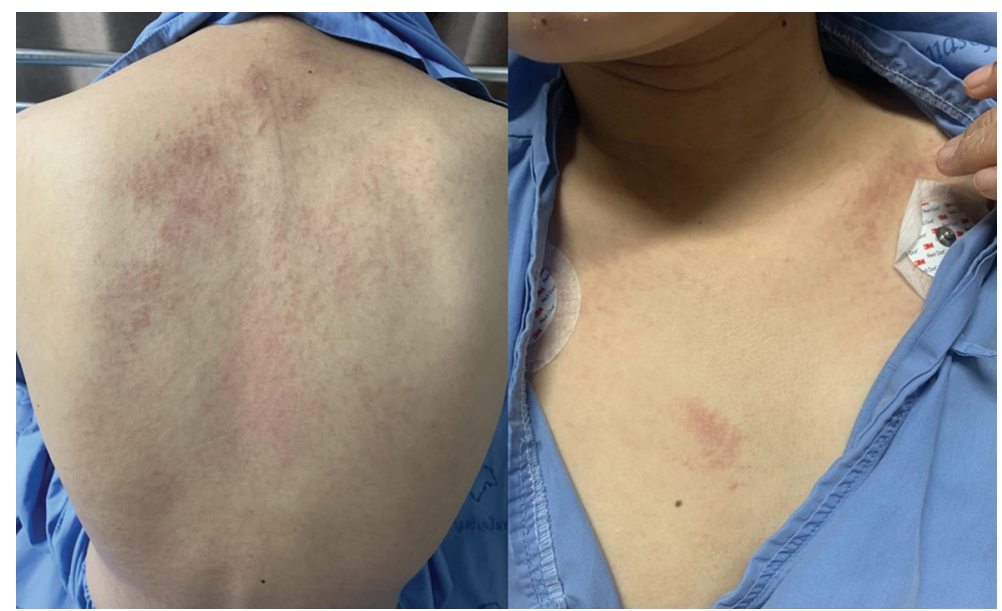

Figure 2. Salmon-pink maculopapular rash on the back and upper chest wall. 
and negative hepatitis $\mathrm{B}$ and $\mathrm{C}$ virus panels. Hemocultures were negative for bacteria, mycobacteria and fungus. Blood complement (C3 and C4) levels were normal. A speckle-pattern antinuclear antibody (ANA) was reported at a low titer of 1:80. Blood test for anti-dsDNA, anti-Sm and anti-cardiolipin antibodies as well as rheumatoid factor were negative.

Computed tomography of the chest and abdomen was performed, revealing an unremarkable results except for a small focal consolidation at the posterior basal segment of the left lower lobe of left lung and minimal left pleural effusion. A bronchoscopy with bronchoalveolar lavage (BAL) was subsequently performed. BAL fluid culture for bacteria, mycobacteria and fungus were negative, as well as the polymerase chain reaction (PCR) for mycobacterium. No malignant cells was found from the BAL fluid cytology study. Trans-bronchial lung biopsy showed non-specific inflammation characterized by benign lung parenchyma and mild interstitial lymphocytic infiltration and fibrosis, without granuloma or malignancy. A skin biopsy at the upper back showed non-specific dermatitis characterized by mild superficial perivascular lymphocytic infiltration in the dermis and few individual necrotic keratinocytes in the epidermis.

After three weeks of inpatient investigation, the patient was diagnosed with AOSD according to both the Yamaguchi's ${ }^{9}$ and Fautrel's classification criteria. ${ }^{10}$ The pulmonary infiltrates and pleuritis was also judged to be associated with AOSD. Oral prednisolone (40 mg daily) was initiated which lead to a dramatic response, characterized by rapid normalization of fever and complete resolution of arthritis and rash within 24 hours (figure 1). At an outpatient visit two weeks after corticosteroid therapy, the patient was free of symptom and her blood tests showed a WBC count of 6,000 cells $/ \mathrm{mm}^{3}$, ferritin of $409 \mathrm{ng} / \mathrm{dL}$ and normal AST and ALT. Immunosuppressive agent was deemed unnecessary in this patient.

\section{Discussion}

Our patient presented with typical features of AOSD: daily spiking fever, polyarthritis, transient salmon-pink rash, leukocytosis, transaminitis and markedly elevated serum ferritin. ${ }^{11}$ Histological findings from the rash of people with AOSD are non-specific, which usually include perivascular infiltration of lymphocytes and/or neutrophils, and necrotic keratinocytes in the superficial epidermis. ${ }^{12}$ The skin histology from our patient was therefore consistent with AOSD. Pulmonary infiltrates accompanied by small amount of pleural effusion are relatively common in AOSD patients, with estimated prevalence of $9 \%$ to $55 \%$ ( $13 \%$ in Thai series). ${ }^{11}$ Histological findings from lung biopsy typically showed changes indicative of acute pneumonitis and focal fibrosis without vasculitis, ${ }^{13}$ which were also consistent with findings in our patient.

SLE was considered a major differential diagnosis in our patient because of the presence of low titer ANA. However, a low titer ANA has been reported in most AOSD series (prevalence $4 \%$ to $13 \%),{ }^{11}$ and ANA at 1:80 titer can also be present in up to $12 \%$ of healthy individuals. ${ }^{14}$ Without any other manifestations suggestive of SLE, the low titer ANA in our patient was therefore considered to have limited diagnostic value.
The diagnosis of AOSD was ultimately made six weeks after the onset of fever.

Clinical presentations in our patient appeared to be similar to the two previous case reports, both of whom had spiking fever, salmon-pink rash, polyarthritis, leukocytosis and elevated serum ferritin. ${ }^{7,8}$ Pleuritis was also report in one case. Both cases however developed symptoms two days after vaccination, compared to seven days in our patient. All three cases did not report any immediate adverse reaction to the vaccine.

Several viral infections, including influenza viruses, have been postulated as possible triggers of AOSD. ${ }^{15}$ Viral proteins could act as danger signals (i.e., pathogen-associated molecular patterns, PAMPs) that initiate the pro-inflammatory cascade via activation of inflammasomes in macrophages and subsequent production of interleukin (IL)-1 $\beta$, IL- 6 and tumor necrosis factor (TNF), all of which are major inflammatory cytokines in AOSD. ${ }^{15}$ Since both viral infections and vaccination could theoretically activate immune response through either bystander activation or molecular mimicry, ${ }^{16}$ it is therefore possible that viral proteins from vaccines could trigger AOSD. There are however insufficient data to support such speculation because AOSD is a rare condition. Furthermore, vaccination has not been traditionally associated with AOSD which could lead to an underestimated prevalence of vaccination-associated AOSD, if such association exists.

We recognize that AOSD in our patient could have been triggered by factors other than influenza vaccination (e.g., rubella, measles, chlamydial infection, solid or hematologic malignancies) that occurred during the seven-day period after receiving the vaccine. ${ }^{1}$ These possible triggers, however, should have been identified during the exhaustive in-hospital investigation. We therefore believe that influenza vaccination was the most likely trigger in our patient, despite the relatively long interval (seven days) between vaccination and the onset of AOSD symptoms.

Although larger number of cases are needed to confirm if influenza vaccination is truly associated with AOSD, we encourage clinicians to explore history of recent vaccination while evaluating people suspected of having systemic inflammatory disorders, including AOSD. In conclusion, we reported a rare case of influenza vaccination-associated AOSD. Our case may help raise awareness of influenza vaccine as possible trigger of AOSD, as well as other systemic inflammatory disorders. However, more studies are needed to confirm the validity of such associations and the immune process behind it.

\section{Ethical approval and informed consent}

Approval from institutional ethics review boards is not required for this type of report (case report involving no more than three participants). Written informed consent for publication was obtained from the patient. Patient data and photos were appropriately de-identified for use in this report.

\section{Conflict of interest}

The author have no conflict of interest relevant to this article. 


\section{Author contribution}

Kanon Jatuworapruk collected the data, obtained the informed consent and drafted the manuscript. The author has reviewed and given approval to the submitted version of the manuscript.

\section{References}

1. Giacomelli R, Ruscitti P, Shoenfeld Y. A comprehensive review on adult onset Still's disease. J Autoimmun. 2018;93:24-36.

2. Mahroum N, Mahagna H, Amital H. Diagnosis and classification of adult Still's disease. J Autoimmun. 2014;48-49:34-7.

3. Liozon E, Parreau S, Filloux M, Dumonteil S, Gondran G, Bezanahary $\mathrm{H}$, et al. Giant cell arteritis or polymyalgia rheumatica after influenza vaccination: A study of 12 patients and a literature review. Autoimmun Rev. 2020:102732.

4. Birck R, Kaelsch I, Schnuelle P, Flores-Suárez LF, Nowack R. ANCA -associated vasculitis following influenza vaccination: causal association or mere coincidence? J Clin Rheumatol. 2009;15(6):289-91.

5. Shimada S, Watanabe T, Sato S. A Patient with Kawasaki Disease Following Influenza Vaccinations. Pediatr Infect Dis J. 2015;34(8):913.

6. Vainer-Mossel ED, Mekori YA, Mor A. Ischemic stroke in a patient with lupus following influenza vaccination: a questionable association. Isr Med Assoc J. 2009;11(3):186-7.

7. Yoo WH. Adult onset Still's disease following influenza vaccination. Joint Bone Spine. 2010;77(4):373-4.
8. Yoshioka K, Fujimoto S, Oba H, Minami M, Aoki T. Onset of adult-onset Still's disease following influenza vaccination. Mod Rheumatol. 2011; 21(4):432-5.

9. Yamaguchi M, Ohta A, Tsunematsu T, Kasukawa R, Mizushima Y, Kashiwagi H, et al. Preliminary criteria for classification of adult Still's disease. J Rheumatol. 1992;19(3):424-30.

10. Fautrel B, Zing E, Golmard JL, Le Moel G, Bissery A, Rioux C, et al Proposal for a new set of classification criteria for adult-onset still disease. Medicine (Baltimore). 2002;81(3):194-200.

11. Louthrenoo W, Aramsareewong T, Sukitawut W. Adult onset Still's disease: clinical features and outcome in 16 Thai patients. J Clin Rheumatol. 2001;7(5):301-7.

12. Lee JY, Hsu CK, Liu MF, Chao SC. Evanescent and persistent pruritic eruptions of adult-onset still disease: a clinical and pathologic study of 36 patients. Semin Arthritis Rheum. 2012;42(3):317-26.

13. Cheema GS, Quismorio FP Jr. Pulmonary involvement in adult-onset Still's disease. Curr Opin Pulm Med. 1999;5(5):305-9.

14. Kavanaugh A, Tomar R, Reveille J, Solomon DH, Homburger HA Guidelines for clinical use of the antinuclear antibody test and tests for specific autoantibodies to nuclear antigens. American College of Pathologists. Arch Pathol Lab Med. 2000;124(1):71-81.

15. Feist E, Mitrovic S, Fautrel B. Mechanisms, biomarkers and targets for adult-onset Still's disease. Nat Rev Rheumatol. 2018;14(10):603-18.

16. Salemi S, D'Amelio R. Could autoimmunity be induced by vaccination? Int Rev Immunol. 2010;29(3):247-69. 